Die Brennstoffzellentechnologie und ihre Nachhaltigkeitspotenziale

\title{
Gretchenfrage Brennstoff(zelle)
}

Brennstoffzellen gelten als Schlüsseltechnologie für mehr Nachhaltigkeit. Allerdings hängen mögliche positive Nachhaltigkeitseffekte dieser Technologie eng mit der Wahl und der Herkunft des Brennstoffs sowie der dazugehörigen Infrastrukfur zusammen. Hieran entscheiden sich ihre ökonomischen und ökologischen Perspektiven sowie das Ausmaß der ausgelösten gesellschaftlichen Veränderungen.

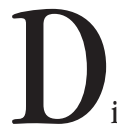

Von Bernd Hirschl und Esther Hoffmann ie Brennstoffzellentechnologie ist seit Mitte der 90er Jahre ein Hoffnungsträger auf eine umweltfreundliche technische Zukunft. Es handelt sich um eine etwa 150 Jahre alte Erfindung, bei der Wasserstoff oder ein wasserstoffhaltiger Brennstoff in einem elektrochemischen Prozess mit Sauerstoff in Verbindung gebracht wird. Als Reaktionsprodukt entsteht hierbei lediglich Wasser, weshalb die Brennstoffzelle zunächst als Antriebsersatz beim Emissions- und Klimasünder Auto als $\mathrm{Zu}$ kunftsvision Einzug hielt - denn sie verspricht „Zero-emission“ während der Fahrt. Die beiden Reaktionsgase sind in der Zelle zunächst durch einen Elektrolyten getrennt, wodurch eine Potenzialdifferenz (elektrische Spannung) entsteht mit der Strom und Wärme produziert werden können.

Das eigentlich ,revolutionäre“ Potenzial dieser Technologie liegt in ihrer Einsatzvielfalt, denn mit der produzierten Energie können verschiedene Anwendungen versorgt werden. Grundsätzlich sind mobile, stationäre und portable Nutzungen möglich. Anwendungsbeispiele sind elektromotorbetriebene Fahrzeuge, Anlagen zur Strom- und Wärmeversorgung im Wohnungs-, Siedlungs- und Prozesswärmebereich sowie elektrische Geräte zum Betrieb von Mobiltelefonen und Notebooks. Zudem lässt sich die Brennstoffzelle mit einer Vielfalt von Brennstoffen betreiben. Aufgrund ihrer Einsatz- und Brennstoffvielfalt gilt die Brennstoffzellentechnologie bei vielen Akteuren als Schlüsseltechnologie, die zukünftig sogar zu einer Basistechnologie werden kann. Von diesen Zielen ist sie gegenwärtig jedoch noch weit entfernt, denn obgleich in den genannten Anwendungsfeldern und auch bei allen Beispielen geforscht und entwickelt wird, ist noch in keinem Fall annähernd Marktreife erreicht.

\section{Wie nachhaltig sind Brennstoffzellen?}

Seit die ersten Brennstoffzellenfahrzeuge in den 90er Jahren vom Daimler-Konzern konzipiert wurden, hat sich in der Branche einiges getan. Es gibt kaum einen internationalen Automobilkonzern, der nicht an der Entwicklung von Brennstoffzellenfahrzeugen arbeitet. Die Hersteller versprechen sich von diesen „umweltfreundlichen" Autos auch in ferner $\mathrm{Zu}$ kunft ökonomische Benefits bei einem durch Brennstoffzellenantriebe weiter wachsenden Automobilmarkt. Unter dem Gesichtspunkt einer nachhaltigen Entwicklung ist dabei problematisch, dass trotz der in Aussicht stehenden technischen Lösung für die lokale Emissionsproblematik der Energieverbrauch pro Auto sowie durch ein insgesamt wachsendes Verkehrsaufkommen weiter steigen kann. Auch die Frage der Brennstoffherkunft, auf die später noch eingegangen wird, ist hierbei noch nicht hinreichend berücksichtigt. So ist für den Verkehrsbereich zu konstatieren, dass die Brennstoffzelle aus unternehmerischer Sicht eine attraktive Zukunftsoption bietet, gesellschaftlich kann sie jedoch für eine Verschlechterung des Status quo sorgen - wenngleich bei wahrscheinlich verbesserter Luftqualität.

Bei den stationären Brennstoffzellen sieht die Situation anders aus. Sie sind als dezentrale Systeme zunächst eine „Bedrohung“ für die etablierten, zentral strukturierten Energieversorger, eröffnen jedoch große volkswirtschaftliche Potenziale und können zu einer gravierenden gesellschaftlichen Veränderung im Bereich der Energieversorgung führen. Der mögliche Wan- del des Energiesektors hin zu mehr Dezentralität verspricht positive volkswirtschaftliche Effekte wie lokale Wertschöpfung, Arbeitsplätze in KMU sowie Handwerk und kann neue, innovative Akteure im Markt stärken. Privatpersonen können vom Energieverbraucher zum -produzenten werden. Die zukünftigen Vertriebsstrukturen sind jedoch noch ebenso unklar wie die Auswirkungen auf das Nutzerverhalten. Schließlich können auch die bestehenden Energieversorgungsunternehmen neue Geschäftsbereiche durch innovative Dienstleistungen wie Contracting erschließen (1). Die ökologischen Vorteile stationärer Brennstoffzellen ergeben sich durch ihre Dezentralität und ihre Effizienz. Im dezentralen Einsatz führen sie zu verringerten Leitungsverlusten. Im Vergleich zu konventionellen Kraft-Wärme-Kopplungs-Technologien bieten sie zudem das Potenzial für höhere Wirkungsgrade, bessere Eigenschaften im Teillastbetrieb sowie eine höhere Stromkennzahl. Mit diesen Vorteilen in allen drei Nachhaltigkeitsdimensionen weist die dezentral-stationäre Anwendung auch als einzige das Potenzial für eine nachhaltige Systeminnovation auf (2).

Letztlich hängt eine Nachhaltigkeitsbewertung generell davon ab, ob die Innovation Brennstoffzelle tatsächlich zu einer Schlüssel- oder Basisinnovation wird, oder ob sie eine Nischenanwendung bleibt. Hier sind in keinem der drei Einsatzbereiche bereits Entscheidungen gefällt oder eindeutige Pfade beschritten worden. Diese hängen wiederum in allen Fällen stark von der entscheidenden ökologischen und wohl auch ökonomischen Frage nach der Wahl der Brennstoffe ab.

\section{Gretchenfrage Brennstoff}

Für den Brennstoffeinsatz lassen sich grundsätzlich zwei Wege unterscheiden. Erstens können Brennstoffzellen mit wasserstoffreichen Brennstoffen (Kohlenwasserstoffe) betrieben werden. Hierbei ist wiederum zu unterscheiden, ob die Zelle mit einem solchen Brennstoff direkt betrieben werden kann, oder ob vorher durch einen Reforming-Prozess der Wasserstoff abgespalten werden muss. Zweitens kann die Zelle auch mit reinem Wasserstoff betrieben werden, der zuvor unter Einsatz von Strom durch Elektrolyse erzeugt und der Zelle über eine geeignete Infrastruktur wie Leitungen und Tanks zugeführt wurde. Für beide Varianten gibt es fossile und regenerative Pfade. 
Als fossile Brennstoffe bieten sich vor allem Erdgas und Methanol an, aber auch ein Betrieb mit Benzin oder Heizöl ist möglich. Als regenerative Brennstoffe sind insbesondere Bioenergieträger wie Bio- und Holzgas, aber auch Sondergase wie Klär-, Deponie- und Grubengas geeignet. Hinzu kommt die Möglichkeit, Wasserstoff mit Hilfe von regenerativem Strom zu erzeugen. Wasserstoff kann langfristig gesehen eine interessante Option für die Speicherung und den Transport von Energie sein. Energieszenarien gehen allerdings davon aus, dass der Bedarf für eine Energielagerung erst bei einem Anteil von 30 Prozent erneuerbare Energien an der Stromerzeugung notwendig ist, was aber nicht vor 2030 realistisch ist (3).

Der Vorteil der Brennstoffzelle liegt darin, dass sie aufgrund der Brennstoffvielfalt einen gleitenden Übergang von fossilen zu regenerativen Brennstoffen unterstützt. Hierbei ist der Brennstoff zur Technologieeinführung im wesentlichen Erdgas, das den Vorteil einer vorhandenen Infrastruktur aufweist. Zudem ist es im Vergleich zu anderen fossilen Brennstoffen emissionsärmer.

Das langfristige Ziel der Entwicklung sollte jedoch unter Nachhaltigkeitsgesichtspunkten die Versorgung von Brennstoffzellen mit biogenen Brennstoffen oder mit regenerativ erzeugtem Wasserstoff sein. Hierbei wirken die Wasserstoffszenarien teilweise wie optimistische großtechnische Visionen. Wasserstoff ist zudem derzeit noch sehr teuer in Herstellung, Lagerung und Transport und birgt dabei teilweise noch erhebliche Risiken und technische Probleme. Darüber hinaus kann freigesetzter Wasserstoff negative Auswirkungen auf die Ozonschicht haben. Demgegenüber sind Bioenergieträger aus ökologischer Sicht deutlich unproblematischer wenngleich hier die Potenziale begrenzter sind und somit die Gefahr massenhaften (Intensiv-) Anbaus bestünde. Zudem befindet sich die technische Entwicklung der Biobrennstoffnutzung noch in den Anfängen.

Es zeigt sich, dass ein derartiger Systemwechsel in seinen Folgen durch umfassendere Technikfolgenabschätzungen antizipiert werden muss - und erst dadurch eine Nachhaltigkeitsbewertung möglich wird (4).

\section{- Fazit}

Die Einführung der Brennstoffzelle weist durchaus in allen Anwendungsbereichen ein Nachhaltigkeitspotenzial auf. Die Ausschöpfung dieses
Potenzials wird jedoch stark durch die Wahl der Brennstoffe beeinflusst. Die Versorgung mit Wasserstoff birgt zahlreiche ökonomische und ökologische Risiken, sodass sie nicht die primär angestrebte - und schon gar nicht die primär geförderte - sein sollte. Zunächst sollte auf der Basis verfügbarer, idealerweise regenerativer Brennstoffe mit guten Infrastrukturvoraussetzungen wie Erdgas, Biogas, Biotreibstoffe eine Einführung erfolgen. Die Entwicklung von wasserstoffbasierten Systemen sollte zunächst weiter erforscht werden, eine breite Einführung in fernerer Zukunft erfordert anspruchsvolle Auflagen für ökologische und sicherheitstechnische Aspekte bei Herstellung, Transport, Lagerung und Gebrauch.

\section{Anmerkungen}

(1) Zu sozialen und ökologischen Auswirkungen stationärer Brennstoffzellen vgl. Hirschl, B./ Hoffmann,

E.: Zukunftstechnologie Brennstoffzelle: Diffusionsbedingungen und sozial-ökologische Forschungsempfehlungen unter besonderer Berücksichtigung dezentraler Energieversorgung. Schriftenreihe IÖW 165/03, Berlin 2003.

(2) Vgl. Konrad, W./ Nill, J.: Innovationen zur Nachhaltigkeit: Ein interdisziplinärer Beitrag zur konzeptionellen Klärung aus wirtschafts- und sozialwissenschaftlicher Perspektive. Schriftenreihe IÖW 157/01, Berlin 2001, sowie Hirschl/Hoffmann a.a.0..

(3) Pehnt, M./ Ramesohl, S.: Fuel cells for distributed power. Benefits, barriers and perspectives. Report commissioned by WWF in co-operation with Fuel Cell Europe. Brussels 2003, www.panda.org/downloads/ europe/stationaryfuelcellsreport.pdf, Zugriff:

14.10.2003.

(4) Zu einer ersten Technikfolgenabschätzung von Brennstoffzellen vgl. Oertel, D./ Fleischer, T.: TA-Projekt „Brennstoffzellen-Technologie”. TAB-Arbeitsbericht Nr. 67, Berlin 2001.

\section{Die Autorlnnen}

Esther Hoffmann ist wissenschaftliche Mitarbeiterin im Forschungsfeld Ökologische Unternehmenspolitik, Bernd Hirschl wissenschaftlicher Mitarbeiter im Forschungsfeld Ökologische Produktpolitik am Institut für ökologische Wirtschaftsforschung (IÖW). Kontakt: IÖW, Potsdamer Str. 105, 10785 Berlin. Tel. 030-8845940, E-Mail: esther.hoffmann@ioew.de, bernd.hirsch@@ioew.de 
(c) 20I0 Authors; licensee IÖW and oekom verlag. This is an article distributed under the terms of the Creative Commons Attribution Non-Commercial No Derivates License (http://creativecommons.org/licenses/by-nc-nd/3.o/), which permits unrestricted use, distribution, and reproduction in any medium, provided the original work is properly cited. 